\begin{tabular}{|c|l|}
\hline Title & The role of tumor necrosis factor-a for interleukin-10 production by murine dendritic cells \\
\hline Author(s) & $\begin{array}{l}\text { Hirata, Noriyuki; Y anagawa, Yoshiki; Ogura, Hisako; Satoh, Masashi; Noguchi, Masay uki; Matsumoto, Machiko; } \\
\text { Togashi, Hiroko; Onoé, Kazunori; Iwabuchi, Kazuya }\end{array}$ \\
\hline Citation & $\begin{array}{l}\text { Cellular Immunology, 266(2), 165-171 } \\
\text { https://doi.org/10.1016/.Cellimm.2010.09.012 }\end{array}$ \\
\hline Issue Date & 2011 \\
\hline Doc URL & http://hdl.handle.net/2115/45137 \\
\hline Type & article (author version) \\
\hline File Information & CI266-2_165-171.pdf \\
\hline
\end{tabular}

Instructions for use 


\section{The role of tumor necrosis factor- $\alpha$ for interleukin-10 production by murine dendritic cells}

Authors: Noriyuki Hirata ${ }^{\mathrm{a}, \mathrm{b}}$, Yoshiki Yanagawa ${ }^{\mathrm{a}, \mathrm{c}, *}$, Hisako Ogura $^{\mathrm{a}}$, Masashi Satoh ${ }^{\mathrm{a}}$, Masayuki Noguchi ${ }^{\mathrm{b}}$, Machiko Matsumoto ${ }^{\mathrm{c}}$, Hiroko Togashi ${ }^{\mathrm{c}}$, Kazunori Onoé ${ }^{\mathrm{a}}$, Kazuya Iwabuchi $^{\mathrm{a}}$

\section{Affiliation:}

${ }^{\text {a }}$ Division of Immunobiology, Institute for Genetic Medicine, Hokkaido University, West 7 North 15, Sapporo 060-0815, Japan

${ }^{\mathrm{b}}$ Division of Cancer Biology, Institute for Genetic Medicine, Hokkaido University, West 7 North 15, Sapporo 060-0815, Japan

${ }^{\mathrm{c}}$ Department of Pharmacology, Faculty of Pharmaceutical Science, Health Sciences University of Hokkaido, Kanazawa 1757, Ishikari-Tobetsu 060-0293, Japan

* Corresponding author. Address: Department of Pharmacology, Faculty of Pharmaceutical Science, Health Sciences University of Hokkaido, Kanazawa 1757, Ishikari-Tobetsu, 060-0293, Japan. Tel. /Fax:+81-133-23-1579. E-mail address: yanagawa@hoku-iryo-u.ac.jp (Y. Yanagawa).

Keywords: Dendritic Cells; Interleukin-10; Signal Transduction; Tumor necrosis factor- $\alpha$; Toll-like receptor 


\section{Abstract}

In the present study, we examined the role of tumor necrosis factor (TNF) in interleukin (IL)-10 production by dendritic cells (DCs) using bone-marrow derived DCs from wild type (WT) and TNF- $\alpha$ knockout (TNF- $\alpha^{-/}$) mice. Toll-like receptor (TLR) stimulation induced substantial level of IL-10 production by WT DCs, but significantly low level of IL-10 production by TNF- $\alpha^{-/-}$DCs. In contrast, no significant difference was detected in IL-12 p40 production between WT and TNF- $\alpha^{-/}$DCs. Addition of TNF- $\alpha$ during TLR stimulation recovered the impaired ability of TNF- $\alpha^{-/}$DCs for IL-10 production. This recovery appeared to be associated with an activation of extracellular signal-regulated kinase, p38 mitogen-activated protein kinase, and phosphatidylinositol 3-kinase/Akt following the TNF- $\alpha$ addition. Blocking these kinases significantly inhibited IL-10 production by TNF- $\alpha^{-1-}$ DCs stimulated with TLR ligands plus TNF- $\alpha$. Thus, TNF- $\alpha$ may be a key molecule to regulate the balance between anti-inflammatory versus inflammatory cytokine production in DCs. 


\section{Introduction}

Tumor necrosis factor (TNF)- $\alpha$ is a multifunctional cytokine that regulates immunity, inflammation, cell differentiation, proliferation, and apoptosis [1,2]. TNF- $\alpha$ binds to two distinct receptors, TNF receptor type 1 (TNFR1; CD120a) and TNF receptor type 2 (TNFR2; CD120b) [3]. Most of TNF functions are thought to be induced via TNFR1. TNFR1 is constitutively expressed in most tissues. TNFR1 recruits death-domain proteins including TNFR1-associated death domain protein (TRADD) and Fas-associated death domain proteins (FADDs) [4]. TRADD activates FADDs, receptor-interacting protein 1 (RIP1), and TNF receptor-associated factor 2 (TRAF2). TRAF2 and RIP1 activate mitogen-activated protein kinases (MAPKs), such as extracellular signal-regulated kinase (ERK), p38 MAPK, c-jun

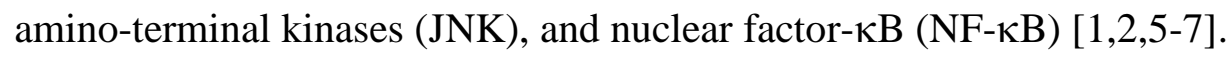

Toll-like receptors (TLRs) are pattern recognition receptors that recognize pathogen-associated molecular patterns (PAMPs) in the pathogen-derived molecules such as lipopolysaccharide (LPS) and induce innate immune responses. The TLR family consists of more than 13 members in mammals [8]. TLRs recognize various PAMPs, such as lipoproteins (TLR2), peptidoglycan (TLR2), double-stranded RNA (TLR3), LPS (TLR4), flagellin (TLR5), single-stranded RNA (TLR7 and TLR8), and CpG-DNA (TLR9). TLRs contain a Toll-IL-1R (TIR) domain [9,10]. Upon TLR activation, TIR domain efficiently recruits several TIR-containing intracellular adaptor proteins including myeloid differentiation primary-response gene 88 (MyD88) [11,12] and TIR domain-containing adaptor inducing interferon (IFN)- $\beta$ (TRIF) $[13,14]$. The MyD88-dependent signaling pathway activates MAPKs, NF-kB, phosphatidylinositol 3-kinase (PI3K)/Akt, activator protein-1, and IFN regulatory factor (IRF) 5 via TRAF6, which induces inflammatory cytokine synthesis. The TRIF-dependent signaling pathway activates IRF3, IRF7, and NF-kB via RIP1, TRAF3, and TRAF6, which induces type I IFNs [10,15-19]. 
Dendritic cells (DCs) are potent professional antigen-presenting cells and play important roles in the initiation and the regulation of immune responses to various pathogenic antigens [20-22]. TLR-stimulated DCs produce a large amount of inflammatory cytokines including TNF- $\alpha$, interleukin (IL)-1, IL-6, and IL-12 and also anti-inflammatory cytokines such as IL-10. DC-produced IL-12 drives polarization of naïve CD4 ${ }^{+}$T cells toward T helper type 1 (Th1) cells, while the IL-10 is involved in inhibition of Th1 responses and differentiation of regulatory T cells [23-26]. It seems that the balance of inflammatory versus anti-inflammatory cytokines produced by DCs is crucial to control immune homeostasis. However, the mechanism underlying regulation of the cytokine balance has not been fully understood.

TNF- $\alpha$ exhibits not only proinflammatory functions but also displays anti-inflammatory properties [27-29]. It has been reported that TNF- $\alpha$ promotes IL-10 production by human monocytes [30,31], although molecular mechanism underlying the TNF- $\alpha$-mediated regulation of IL-10 production remains unclear. On the other hand, the role of TNF- $\alpha$ for IL-10 production by DCs has not been well documented. In the present study, using bone-marrow derived DCs (BMDCs) generated from either wild type (WT) or TNF- $\alpha$ knockout (TNF- $\alpha^{-/}$) mice, we examined the role of TNF- $\alpha$ in DC production of IL-10 upon TLR stimulation focusing on the intracellular signaling.

\section{Materials and Methods}

\subsection{Mice}

WT C57BL/6 mice were purchased from Japan SLC (Hamamatsu, Japan). TNF- $\alpha$ knockout $\left(\mathrm{TNF}-\alpha^{-/-}\right.$) mice with C57BL/6 background were obtained from The Jackson Laboratory (Bar Harbor, ME). All mice were maintained in a specific pathogen-free condition of our animal facility at Hokkaido University. Female mice at 6 to 14-weeks old were used for the 
preparation of bone marrow cells. All experiments were approved by regulations of Hokkaido University Animal Care and Use Committee.

\subsection{Reagents and antibodies (Abs)}

Murine recombinant granulocyte-macrophage colony-stimulating factor (GM-CSF) and murine recombinant TNF- $\alpha$ were purchased from PeproTech (Rocky Hill, NJ). Rabbit complement was purchased from Cedarlane (Ontario, Canada). LPS (ultra-pure grade) from Escherichia coli (O111:B4) and Pam3CSK4 (P3C), a synthetic lipopeptide, were purchased from Invivogen (San Diego, CA). Fluorescein isothiocyanate (FITC)-conjugated anti-mouse CD86 monoclonal Ab (mAb) (GL1), phycoerythrin (PE)-conjugated anti-mouse CD40 mAb (3/23), biotin-conjugated anti-I-A ${ }^{b}$ mAb (AF6-120.1), and streptavidin-peridinin chlorophyll protein (PerCP) were obtained from BD Pharmingen (San Jose, CA). Anti-mouse TNF- $\alpha$ mAb (MP6-XT22), anti-mouse TNFR1 mAb (55R-170), and anti-mouse TNFR2 mAb (TR75-54.7) were purchased from Biolegend (San Diego, CA). Anti-phospho-ERK1/2 $\left(\mathrm{Thr}^{202} / \mathrm{Tyr}^{204}\right.$ ) mAb (197G2), anti-phospho-p38 MAPK (Thr ${ }^{180} / \mathrm{Tyr}^{182}$ ) Ab, anti-phospho-JNK1/2 (Thr ${ }^{183} / \mathrm{Tyr}^{185}$ ) mAb (G9), anti-phospho-Akt (Ser ${ }^{473}$ ) mAb (193H12), and anti-GAPDH mAb (14C10) were purchased from Cell Signaling Technology (Beverly, MA). U0126, a specific inhibitor of MAPK/ERK kinase (MEK)1/2, and SB203580, a specific inhibitor of p38 MAPK, were purchased from Calbiochem (San Diego, CA). LY294002, a specific inhibitor of PI3K, was purchased from Sigma-Aldrich (St. Louis, MO).

\subsection{Generation of BMDCs}

Murine BMDCs were generated by a well established method as previously described [32,33] with a minor modification. Bone marrow cells were prepared from femur and tibial bone marrow of WT or TNF- $\alpha^{-/-}$mice. After lysis of erythrocytes, major histocompatibility 
complex class II-, CD45R (B220)-, CD4-, and CD8-positive cells were removed by killing with a cocktail of relevant mAbs (1E4, RA3-6B2, GK1.5, and 53-6.7) and rabbit complement. The cells were extensively washed to remove mAbs, complement, and cell debris. The cells were cultured in RPMI-1640 containing 5\% fetal calf serum (FCS) and GM-CSF (20 ng/ml) at a density of $1 \times 10^{6}$ cells $/ \mathrm{ml} /$ well (24-well plate). On day 2, the medium was gently exchanged to fresh medium. On day 4, non-adherent granulocytes were removed without dislodging clusters of developing DCs, and fresh medium was added. On day 6, free-floating and loosely adherent cells were collected and were used as BMDCs (>95\% CD11c ${ }^{+}$B220 $)$.

\subsection{Measurement of cytokines in culture supernatants}

BMDCs $\left(2 \times 10^{5} / \mathrm{ml}\right)$ were treated with TNF- $\alpha$ and/or P3C $(100 \mathrm{ng} / \mathrm{ml})$ plus LPS $(1 \mu \mathrm{g} / \mathrm{ml})$ (This combination of TLR ligands will be referred to as TLR-L) for $24 \mathrm{~h}$ in 5\% FCS RPMI-1640 [34]. TNF- $\alpha$ was used at $100 \mathrm{ng} / \mathrm{ml}$ based on our preliminary dose-response study (data not shown). In some experiments, cells $\left(2 \times 10^{5} / \mathrm{ml}\right)$ were pretreated with U0126, SB203580, LY294002, or vehicle alone (0.1\% DMSO) for $1 \mathrm{~h}$ and then stimulated with TNF- $\alpha$ and/or TLR-L for $24 \mathrm{~h}$ in the presence of each inhibitor. U0126, SB203580, and LY294002 were used at 10, 30, and $10 \mu \mathrm{M}$, respectively, as previously described [34]. The culture supernatants were subjected to quantification of the protein level of IL-10, IL-12 p40, and TNF- $\alpha$ by enzyme linked immunosorbent assay (ELISA) using OptEIA Set (BD Pharmingen).

\subsection{Immunoblotting}

BMDCs $\left(5 \times 10^{5} / \mathrm{ml}\right)$ were treated with TLR-L and/or TNF- $\alpha$ for $24 \mathrm{~h}$ in $5 \%$ FCS RPMI-1640 for the indicated time period. Reactions were halted by rapidly cooling on ice, and these cells were washed by ice-cold phosphate-buffered saline. The whole cell lysates were prepared using cell lysis buffer (Cell signaling Technology). The cell lysates were separated by 
sodium dodecyl sulfate-polyacrylamide gel electrophoresis, and then blotted onto a polyvinylidene fluoride membrane (Millipore, Bedford, MA). The membrane were proved with primary Ab, and developed with horseradish peroxidase-conjugated secondary Ab by enhanced chemiluminescence.

\subsection{Flow cytometry}

Cell staining with FITC-, PE-, or biotin-conjugated mAb, and streptavidin-PerCP and flow cytometric analysis was performed on EPICS XL (Beckman coulter Inc., Miami, FL) as previously described [35].

\subsection{Statistical analysis}

The Student's $t$-test or Dunnet’s test was used to analyze data for significant differences. $P$ values less than 0.05 were regarded as significant.

\section{Results}

\subsection{Cytokine production by WT and TNF- $\alpha^{-/-}$DCs upon TLR stimulation}

We examined the role of endogenous TNF- $\alpha$ in IL-10 production by murine BMDCs in response to TLR ligands comparing BMDCs from TNF- $\alpha^{-/-}$mice and those from WT mice. We have previously demonstrated that LPS (ultra-pure grade) or P3C (a synthetic TLR2 ligand) alone induced negligible production of IL-10 in BMDCs, while the simultaneous treatment with these ligands results in the vigorous production of IL-10 [34]. Thus, DCs were stimulated with LPS plus P3C to evaluate the ability for the IL-10 production.

BMDCs from WT or TNF- $\alpha^{-/-}$mice were treated with TLR-L $(1 \mu \mathrm{g} / \mathrm{ml}$ LPS plus $100 \mathrm{ng} / \mathrm{ml}$ P3C) for $24 \mathrm{~h}$, and levels of IL-10, IL-12 p40, and TNF- $\alpha$ in the culture supernatants were determined by ELISA (Fig. 1A). Consistent with our previous study, TLR-L induced 
substantial level of IL-10 production by WT DCs. Notably, IL-10 production by TNF- $\alpha^{-/-}$DCs stimulated with TLR-L was significantly suppressed as compared to that by WT DCs (Fig. 1A, left). In contrast, both WT DCs and TNF- $\alpha^{-/-}$DCs vigorously produced IL-12 p40 in response to TLR-L and no significant difference was detected in this cytokine production between these types of DCs (Fig. 1A, center). WT DCs markedly produced TNF- $\alpha$ in response to TLR-L, while TNF- $\alpha^{-/-}$DCs showed no TNF- $\alpha$ production irrespective of the TLR stimulation (Fig. 1A, right). We also analyzed IL-12 p70 production by WT and TNF- $\alpha^{-/-D C s ~ u p o n ~ T L R ~ s t i m u l a t i o n . ~}$ However, no IL-12 p70 production was detected in any cultures tested $(<30$ pg/ml, data not shown). No significant difference was detected in the cell viability between WT and TNF- $\alpha^{-/-}$ DCs after 24 h of culture (data not shown).

\subsection{The effect of exogenous TNF- $\alpha$ in IL-10 production by DCs upon TLR stimulation}

Above findings (Fig. 1A) suggest that DC production of TNF- $\alpha$ lead to the IL-10 production by these DCs in response to TLR ligands. We next examined whether addition of exogenous TNF- $\alpha$ during the TLR stimulation recovered the impaired ability of TNF- $\alpha^{-/-}$DCs to produce IL-10.

BMDCs from WT or TNF- $\alpha^{-/-}$mice were stimulated with TLR-L in the presence or absence of TNF- $\alpha$ (100 ng/ml) for $24 \mathrm{~h}$, and levels of IL-10 and IL-12 p40 in the culture supernatants were determined by ELISA (Fig. 1B). TNF- $\alpha$ alone never induced IL-10 production by both types of DCs (Fig. 1B left). Again, TLR-L induced vigorous production of IL-10 by WT DCs but not by TNF- $\alpha^{-/-}$DCs. Addition of TNF- $\alpha$ restored the TLR-mediated IL-10production by TNF- $\alpha^{-/-}$DCs to the level of that by WT DCs. In contrast, TNF- $\alpha$ showed no significant effect on TLR-L mediated IL-10 production by WT DCs. Thus, TNF- $\alpha$ produced by WT DCs may be necessary to induce maximal IL-10 production by the DCs in response to TLR-L. 
TLR-L markedly induced IL-12 p40 production by WT DCs and TNF- $\alpha^{-/-}$DCs, and no significant difference was detected in the level of IL-12 p40 production between these types of DCs (Fig. 1B, middle). Exogenous TNF- $\alpha$ never affected the TLR-mediated IL-12 p40 production by WT DCs. Although TNF- $\alpha$ slightly increased the TLR-mediated IL-12 p40 production by TNF- $\alpha^{-/-}$DCs, the effect was statistically not significant.

\subsection{Cell surface expressions of maturation markers on TLR-stimulated DCs}

We next analyzed the cell surface expression of several maturation markers on WT and TNF- $\alpha^{-/-}$DCs. WT and TNF- $\alpha^{-/-}$DCs were treated with TLR-L for $24 \mathrm{~h}$ and the cell surface expressions of CD40, CD86, and I-A ${ }^{\mathrm{b}}$ were determined by flow cytometry (Fig. 2). After the TLR stimulation, WT and TNF- $\alpha^{-/-}$DCs exhibited mature phenotype, high expression of CD86, CD40, and I-A $\mathrm{A}^{\mathrm{b}}$. No significant difference was detected in the expression level of these maturation markers between WT and TNF- $\alpha^{-/-}$DCs, although the proportion of CD86 positive cells was increased in TNF- $\alpha^{-/-}$DCs compared to WT DCs (Fig. 2). Addition of TNF- $\alpha$ during the TLR stimulation showed no effect on the level of CD86, CD40, and I-A $\mathrm{A}^{\mathrm{b}}$ expressions in both types of DCs (data not shown). From these results, TNF- $\alpha$ may be dispensable for the phenotypic maturation of DCs in response to TLR stimulation.

\subsection{The effect of TNF- $\alpha$ on activation of Akt and MAPKs in DCs upon TLR stimulation}

PI3K/Akt and MAPK pathways are responsible for production of various cytokines following TLR stimulation [36]. It has been reported that TNF- $\alpha$ is capable of activating these intracellular pathways $[37,38]$. TNF- $\alpha$ promoted IL-10 production in TNF- $\alpha^{-/-}$DCs but not in WT DCs (Fig. 1). To analyze the molecular mechanism responsible for the TNF- $\alpha$-mediated promotion of IL-10 production seen in TNF- $\alpha^{-/-}$DCs, we examined the effect of TNF- $\alpha$ on 
activation of Akt and MAPKs (ERK1/2, p38 MAPK, and JNK1/2) in the TNF- $\alpha^{-/-}$DCs upon TLR stimulation.

BMDCs from TNF- $\alpha^{-/-}$mice were stimulated with TNF- $\alpha$ and/or TLR-L for indicated time periods, and intracellular protein levels of phospho-Akt (pAkt), phospho-ERK1/2 (pERK1/2), phospho-p38 MAPK (pp38 MAPK), and phospho-JNK1/2 (pJNK1/2) were determined by immunoblotting (Fig. 3).

TNF- $\alpha$ markedly increased the level of pERK1/2 in TNF- $\alpha^{-/-}$DCs at 7 min, while showed only slight effect at 15 to 30 min (Fig. 3 A, B). On the other hand, TLR-L induced slight phosphorylation of ERK1/2 in TNF- $\alpha^{-/-}$DCs at 7 min, but markedly increased the level at 15 min. The effect was decreased at $30 \mathrm{~min}$. The pERK1/2 in TNF- $\alpha^{-/-}$DCs at 7 min after treatment with both TLR-L and TNF- $\alpha$ showed same level as that in TNF- $\alpha^{-/-}$DCs treated with TNF- $\alpha$ alone. Thus, the ERK1/2 activation at 7 min seemed to be largely dependent on TNF- $\alpha$. At 15 and 30 min no difference was detected in the pERK1/2 level between TLR-L-treated and TLR-L plus TNF- $\alpha$-treated TNF- $\alpha^{-/-}$DCs.

The levels of pp38 MAPK and pAkt in TNF- $\alpha^{-/-}$DCs were markedly increased at 7 min, and then gradually reduced at 15 to 30 min after TNF- $\alpha$ stimulation (Fig. 3 A, C, E). In contrast, TLR-L increased the levels of these molecules slightly at 7 min, but markedly at 15 and 30 min. Treatment of these DCs with both TNF- $\alpha$ and TLR-L considerably increased the levels of pp38 MAPK and pAkt at 7 min as compared with those in DCs treated with TLR-L alone. Thus, exogenous TNF- $\alpha$ was responsible for an early (7 min) activation of p38 MAPK and Akt.

The level of pJNK1/2 in TNF- $\alpha^{-/-}$DCs was modestly increased at 7 to 15 min and then decreased at 30 min after TNF- $\alpha$ stimulation (Fig. 3 A, D). In contrast, TLR-L increased the level of pJNK1/2 modestly at 7 min and markedly at 15 and 30 min. Exogenous TNF- $\alpha$, however, showed no significant effect on the TLR-L-mediated phosphorylation of pJNK1/2 at 
all time periods tested.

3.5. The effect of ERK1/2, p38 MAPK, or PI3K inhibition on IL-10 and IL-12 p40 production by TNF- $\alpha^{-/-}$DCs upon TNF- $\alpha$ plus TLR-L stimulation

ERK1/2, p38 MAPK, and Akt activation in TNF- $\alpha^{-/-}$DCs upon TLR stimulation was significantly enhanced by addition of TNF- $\alpha$ at an early time point (Fig. 3). We thus examined the role of ERK1/2, p38 MAPK, and Akt in IL-10 production by TNF- $\alpha^{-/-}$DCs upon stimulation with TLR-L plus TNF- $\alpha$ using U0126, a specific inhibitor of MEK-ERK pathway, SB203580, a specific inhibitor of p38 MAPK, and LY294002, a specific inhibitor of PI3K.

TNF- $\alpha^{-/-}$DCs were pretreated with U0126, SB203530, LY294002, or vehicle alone $(0.1 \%$ DMSO) for $1 \mathrm{~h}$ and then stimulated with TNF- $\alpha$ and/or TLR-L for $24 \mathrm{~h}$ in the presence of each inhibitor. The amount of IL-10 and IL-12 p40 in the culture supernatant was quantitated by ELISA (Fig. 4). Again, stimulation with TLR-L plus TNF- $\alpha$ induced vigorous production of IL-10 and IL-12 p40. U0126 modestly inhibited the IL-10 production and increased the IL-12 p40 production by TNF- $\alpha^{-/-}$DCs. Notably, both SB203580 and LY294002 markedly inhibited the IL-10 production by TNF- $\alpha^{-/-}$DCs upon TLR-L plus TNF- $\alpha$ stimulation, while showing no significant effect on the IL-12 p40 production. Thus, the TNF- $\alpha$-mediated early activation of ERK1/2, p38 MAPK, and PI3K/Akt may be responsible for the promotion of IL-10 production by TNF- $\alpha^{-/-}$DCs upon TLR stimulation.

\section{Discussion}

TLR ligands induce vigorous production of pro-inflammatory cytokines by macrophages and DCs. These TLR-mediated innate immune responses are crucial to initiates acquired immunity. TLR ligands also induce production of IL-10, an anti-inflammatory cytokine, which may suppress undesirable and/or exceeded inflammatory responses. Although transcription factors 
and signal transduction pathways for production of pro-inflammatory cytokines have been well identified, those of anti-inflammatory cytokines such as IL-10 have been elusive. In the present study, we examined the role of TNF- $\alpha$ in DC production of IL-10 upon TLR4 and TLR2 stimulation (TLR4,2 stimulation) and pursued mechanism underlying the TNF- $\alpha$-mediated regulation of IL-10 production. We demonstrated herein that TNF- $\alpha$ was involved in DC production of IL-10 but not IL-12 p40 upon TLR4,2 stimulation. It was also shown that the TNF- $\alpha$-mediated activation of MAPK and PI3K/Akt pathways may be responsible for the TNF- $\alpha$-mediated regulation of IL-10 production.

First, we compared productions of IL-10 and IL-12 p40 between WT and TNF- $\alpha^{-/-}$DCs in response to TLR-L (LPS plus P3C). Upon TLR4,2 stimulation, TNF- $\alpha^{-/-}$DCs produced lower level of IL-10 than those by WT DCs. In contrast, no significant differences were noted in the TLR-mediated IL-12 p40 production between these types of DCs. Addition of TNF- $\alpha$ during the TLR stimulation recovered the impaired ability of TNF- $\alpha^{-/-}$DCs for IL-10 production, although TNF- $\alpha$ alone exerted negligible effect on the IL-10 production. It seems that TNF- $\alpha$ is involved in the enhancement of TLR-mediated production of IL-10, while this cytokine-mediated signaling alone is insufficient to induce a substantial level of IL-10 production by DCs.

We also analyzed the expression of maturation markers, CD86, CD40, and I-A ${ }^{\mathrm{b}}$, on WT and TNF- $\alpha^{-/-}$DCs upon TLR4,2 stimulation. No significant difference was detected in the level of mean fluorescence intensity of these expressions between WT and TNF- $\alpha^{-/-}$DCs. However, the proportion of CD86 positive cells was increased in TNF- $\alpha^{-/-}$DCs compared to WT DCs. Thus, TNF- $\alpha$ may play a role in the regulation of CD86 expression on DCs.

MAPKs and PI3K/Akt are involved in activation of various transcription factors that promote the IL-10 synthesis in macrophages/monocytes and DCs upon TLR stimulation [36, 39, 
40]. Recently, we demonstrated that MAPK and PI3K/Akt pathways were responsible for the promotion of IL-10 production by BMDCs upon TLR stimulation [34]. It has been reported that TNF- $\alpha$ also activates MAPK and PI3K signaling pathways via TNFR1 [1,2,5-7,37,38]. To explore the mechanism underlying the TNF- $\alpha$-mediated regulation of IL-10 production by DCs, we analyzed effect of TNF- $\alpha$ on the activity of MAPKs and PI3K in TNF- $\alpha^{-/-}$DCs upon TLR stimulation. TNF- $\alpha$ markedly enhanced activities of ERK, p38 MAPK, and Akt in TNF- $\alpha^{-/-}$ DCs upon TLR4,2 stimulation at an early phase (at $7 \mathrm{~min}$ ). Blocking the activation of ERK, p38 MAPK, or PI3K significantly inhibited IL-10 production by TNF- $\alpha^{-/-}$DCs upon stimulation with TNF- $\alpha$ plus TLR-L. Thus, TNF- $\alpha$ appears to facilitate the TLR-mediated IL-10 production through the activation of ERK, p38 MAPK, and PI3K/Akt pathways in DCs.

It has been reported that p38 MAPK and Akt are activated via RIP1, while JNK was activated via TRAF2 in TNF- $\alpha$-mediated cellular responses [6]. Thus, we speculate that RIP1 is crucial for the TNF- $\alpha$-mediated effect on IL-10 production in TNF- $\alpha^{-/-}$DCs. On the other hand, Vivarelli et al showed that RIP1 is also involved in TLR4-mediated activation of PI3K-Akt pathway [41]. Thus, TNF- $\alpha$ - and TLR-mediated signaling pathways appear to share the RIP1. Consequently, we could not clarify whether the decrease in IL-10 production in response to MAPK and PI3K inhibitors (Fig. 4) is due to specific inhibition of the TNF- $\alpha$-mediated signaling. Nonetheless, we believe that our present findings are important to understand a regulation system of IL-10 in DCs in response to TLR stimulation.

Recently, several studies also showed that MAPK- or PI3K-mediated signal is involved in IL-10 production and autocrine IL-10 negatively regulated IL-12 production upon TLR stimulation in macrophages and DCs [40-43]. On the other hand, we have previously reported that LY294002 (a PI3K specific inhibitor) decreases IL-10 production, but shows no effect on IL-12 p40 production upon TLR-stimulation in BMDCs [34]. At present, decrease in IL-10 production by inhibiting PI3K activity showed no significant effect on IL-12 p40 production by 
TNF- $\alpha^{-/-}$DCs stimulated with TLR-L plus TNF- $\alpha$ (Fig. 4). The discrepancy between above studies in the autocrine inhibitory effect of IL-10 on IL-12 p40 production may be attributable to the differences in cell types and/or culture conditions such as cell density and stimuli.

We performed the blocking study using anti-TNF- $\alpha$ mAb (clone: MP6-XT22), anti-TNFR1 mAb (clone: 55R-170), and anti-TNFR2 mAb (clone: TR75-54.7). TNF- $\alpha$-induced increase in CD86 expression on BC1 cells [35] was completely inhibited by treatment with the anti-TNFR1 mAb or the anti-TNF- $\alpha$ mAb (data not shown). However, these mAbs failed to exert significant effects on the IL-10 production by DCs upon the TLR stimulation (data not shown). As a possible explanation for the discrepant results, attenuated TNF- $\alpha$ signal in the presence of the mAb might be enough to enhance the TLR-mediated IL-10 production.

Alternatively, a constitutive weak TNF- $\alpha$ signaling before the antibody treatment might be sufficient to facilitate IL-10 production upon TLR stimulation.

We also analyzed production of IL-23, a member of the IL-12 cytokine family, in WT and TNF- $\alpha^{-/-}$DCs upon TLR stimulation. TLR ligands induced substantial production of IL-23 in WT and TNF- $\alpha^{-/-}$DCs. The IL-23 production by TNF- $\alpha^{-/-}$DCs was higher than that by WT DCs (data not shown). In contrast, the IL-12 p40 production upon the TLR stimulation slightly decreased in TNF- $\alpha^{-/-}$DCs compared to WT DCs (Fig. 1). Thus, the level of IL-12 p40 production did not reflect to that of IL-23 production in our culture system. It has been shown that IL-12 p40 expression was not parallel to IL-23 p19 expression in DCs [44]. It seems to us that this point should be carefully analyzed in future studies.

We demonstrated herein that TNF- $\alpha$ signaling to promote DC production of anti-inflammatory cytokine, IL-10, but not inflammatory cytokine, IL-12, upon TLR stimulation. Thus, TNF- $\alpha$ may be a key molecule to regulate the balance between anti-inflammatory versus inflammatory cytokines in DCs. However, the precise mechanism underlying the TNF- $\alpha$-mediated regulation of IL-10 production remains to be unclear. Thus, 
we would like to identify the molecular mechanisms responsible for the TNF- $\alpha$-mediated regulation system in the future studies. Since the balance between IL-10 and IL-12 production by DCs is crucial to induce appropriate immune responses, further elucidation of the TNF- $\alpha$-mediated immune regulation may lead to the development of clinical applications for the treatment of various immune disorders. 


\section{Acknowledgements}

This study was supported in part by a Grant-in-Aid for Scientific Research (B) from the

Japan Society for the Promotion of Science (JSPS), Global COE Program 'Establishment of International Collaboration Center for Zoonosis Control' from Ministry of Education, Japan. 


\section{References}

[1] B.B. Aggrawal, Signalling pathways of the TNF superfamily: a double-edged sword, Nat. Rev. Immunol. 3 (2003) 745-756.

[2] F. Balkwill, Tumour necrosis factor and cancer, Nat. Rev. Cancer. 9 (2009) 361-371.

[3] P. Vandenabeele, W. Declercq, R. Beyaert, W. Fiers, Two tumour necrosis factor receptors: structure and function, Trends Cell Biol. 5 (1995) 392-399.

[4] V. Baud, M. Karin, Signal transduction by tumor necrosis factor and its relatives, Trends Cell Biol. 11 (2001) 372-377.

[5] B.W. Winston, D.W. Riches, Activation of $\mathrm{p} 42^{\text {mapk/erk2 }}$ following engagement of tumor necrosis factor receptor CD120a (p55) in mouse macrophages, J. Immunol. 155 (1995) $1525-1533$.

[6] H. Wajant, K. Pfizenmaier, P. Scheurich, Tumor necrosis factor signaling, Cell Death Differ. 10 (2003) 45-65.

[7] I.E. Wertz, V.M. Dixit, Ubiquitin-mediated regulation of TNFR1 signaling, Cytokine Growth Factor Rev. 19 (2008) 313-324.

[8] T. Kawai, S. Akira, Pathogen recognition with Toll-like receptors, Curr. Opin. Immunol. 17 (2005) 338-344.

[9] J.L. Imler, J.A. Hoffmann, Toll signaling: the TIReless quest for specificity, Nat. Immunol. 4 (2003) 105-106.

[10] L.A. O’Neill, A.G. Bowie, The family of five: TIR-domain-containing adaptors in Toll-like receptor signaling, Nat. Rev. Immunol. 7 (2007) 353-364.

[11] R. Medzhitov, P. Preston-Hurlburt, E. Kopp, A. Stadlen, C. Chen, S. Ghosh, C.A.J. Janeway, MyD88 is an adaptor protein in the hToll/IL-1 receptor family signaling pathways, Mol. Cell. 2 (1998) 253-258.

[12] S. Akira, K. Takeda, T. Kaisho, Toll-like receptors: critical proteins linking innate and 
acquired immunity, Nat. Immunol. 2 (2001) 675-680.

[13] K.A. Fitzgerald, E.M. Palsson-McDermott, A.G. Bowie, C.A. Jefferies, A.S. Mansell, G. Brady, E. Brint, A. Dunne, P. Gray, M.T. Harte, D. McMurray, D.E. Smith, J.E. Sims, T.A. Bird, L.A. O’Neill, Mal (MyD88-adapter-like) is required for Toll-like receptor-4 signal transduction, Nature 413 (2001) 78-83.

[14] T. Horng, G.M. Barton, R. Medzhitov, Tirap: an adapter molecule in the Toll signaling pathway, Nat. Immunol. 2 (2001) 835-841.

[15] M.W. Covert, T.H. Leung, J.E. Gaston, D. Baltimore, Achiving stability of lipopolysaccharide-induced NF-кB activation, Science 309 (2005) 1854-1857.

[16] T. Kawai, S. Akira, Signaling to NF-кB by Toll-like receptors, Trends Mol. Med. 13 (2007) 460-469.

[17] W.L. Yang, J. Wang, C.H. Chan, S.W. Lee, A.D. Campos, B. Lamothe, L. Hur, B.C. Grabiner, X. Lin, B.G. Darnay, H.K. Lin, The E3 ligase TRAF6 regulates Akt ubiquitination and activation, Science 325 (2009) 1134-1138.

[18] S.S. Sierra, S.D. Deshmukh, J. Kalnitski, P. Küenzi, M.P. Wymann, D.T. Golenbock, P. Henneke, Mal connects TLR2 to PI3Kinase activation and phagocyte polarization, EMBO J. 28 (2009) 2018-2027.

[19] M.H. Laird, S.H. Rhee, D.J. Perkins, A.E. Medvedev, W. Piao, M.J. Fenton, S.N. Vogel, TLR4/MyD88/PI3K interactions regulate TLR4 signaling, J. Leukoc. Biol. 85 (2009) 966-977

[20] R.M. Steinman, The dendritic cell system and its role in immunogenicity, Annu. Rev. Immunol. 9 (1991) 271-296.

[21] D.N.J Hart, Dendritic cells: unique leukocyte populations which control the primary immune response, Blood 90 (1997) 3245-3287.

[22] J. Banchereau, R.M. Steinman, Dendritic cells and the control of immunity, Nature 392 
(1998) 245-251.

[23] P. McGuirk, K.H. Mills, Pathogen-specific regulatory T cells provoke a shift in the Th1/Th2 paradigm in immunity to infectious diseases, Trends Immunol. 23 (2002) 450-455.

[24] S. Rutella, S. Danese, G. Leone, Tolerogenic dendritic cells: cytokine modulation comes of age, Blood 108 (2006) 1435-1440.

[25] Y. Belkaid, Regulatory T cells and infection: a dangerous necessity, Nat. Rev. Immunol. 7 (2007) 875-888.

[26] A. O’Garra, P. Vieira, Th1 cells control themselves by producing interleukin-10, Nat. Rev. Immunol. 7 (2007) 425-428.

[27] J. Hodge-Dufour, M.W. Marino, M.R. Horton, A. Jungbluth, M.D. Burdick, R.M. Strieter, P.W. Noble, C.A. Hunter, E. Puré, Inhibition of interferon $\gamma$ induced interleukin 12 production: a potential mechanism for the anti-inflammatory activities of tumor necrosis factor, Proc. Natl. Acad. Sci. USA 95 (1998) 13806-13811.

[28] X. Ma, J. Sun, E. Papasavvas, H. Riemann, S. Robertson, J. Marshall, R.T. Bailer, A. Moore, R.P. Donnelly, G. Trinchieri, L.J. Montaner, Inhibition of IL-12 production in human monocyte-derived macrophages by TNF, J. Immunol. 164 (2000) 1722-1729.

[29] M. Zakharova, H.K. Ziegler, Paradoxical anti-inflammatory actions of TNF- $\alpha$ : Inhibition of IL-12 and IL-23 via TNF Receptor 1 in macrophages and dendritic cells, J. Immunol. 175 (2005) 5024-5033.

[30] C. Wanidworanun, W. Strober, Predominant role of tumor necrosis factor- $\alpha$ in human monocyte IL-10 synthesis, J. Immunol. 151 (1993) 6853-6861.

[31] S.L. Parry, M. Sebbag, M. Feldmann, F.M. Brennan, Contact with T cells modulates monocyte IL-10 production. J. Immunol, 158 (1997) 3673-3681.

[32] K. Inaba, M. Inaba, N. Romani, H. Aya, M. Deguchi, S. Ikehara, S. Muramatsu, R.M. 
Steinman, Generation of large numbers of dendritic cells from mouse bone marrow cultures supplemented with granulocyte/macrophage colony-stimulating factor, J. Exp. Med. 176 (1992) 1693-1702.

[33] S. Yamazaki, A.J. Bonito, R. Spisek, M. Dhodapkar, K. Inaba, R.M. Steinman. Dendritic cells are specialized accessory cells along with TGF- $\beta$ for the differentiation of Foxp3 ${ }^{+}$ $\mathrm{CD}^{+}$reguratory T cells from peripheral Foxp3 precursors, Blood 110 (2007) 4293-4302.

[34] N. Hirata, Y. Yanagawa, T. Ebihara, T. Seya, S. Uematsu, S. Akira, F. Hayashi, K. Iwabuchi, K. Onoé, Selective synergy in anti-inflammatory cytokine production upon cooperated signaling via TLR4 and TLR2 in murine conventional dendritic cells, Mol. Immunol. 45 (2008) 2734-2742.

[35] Y. Yanagawa, N. Iijima, K. Iwabuchi, K. Onoé, Activation of extracellular signal-related kinase by TNF- $\alpha$ controls the maturation and function of murine dendritic cells, J. Leukoc. Biol. 71 (2002) 125-132.

[36] S.M. Mäkelä, M. Strengell, T.E. Pietilä, P. Österlund, I. Julkunen, Multiple signaling pathways contribute to synergistic TLR ligand-dependent cytokine gene expression in human monocyte-derived macrophages and dendritic cells, J. Leukoc. Biol. 85 (2009) $664-672$.

[37] R. Pincheira, A.F. Castro, O.N. Ozes, P.S. Idumalla, D.B. Donner, Type 1 TNF receptor forms a complex with and uses Jak2 and c-Src to selectively engage signaling pathways that regulate transcription factor activity, J. Immunol. 181 (2008) 1288-1298.

[38] M.A. Rivas, R.P. Carnevale, C.J. Proietti, C. Rosemblit, W. Beguelin, M. Salatino, E.H. Charreau, I. Frahm, S. Sapia, P. Brouckaert, P.V. Elizalde, R. Schillaci, TNF $\alpha$ acting on TNFR1 promotes breast cancer growth via p42/P44 MAPK, JNK, Akt and NF-кB-dependent pathways, Exp. Cell. Res. 314 (2008) 509-529.

[39] Y.W. Liu, C.C. Chen, H.P. Tseng, W.C. Chang. Lipopolysaccharide-induced 
transcriptional activation of interleukin-10 is mediated by MAPK- and NF-кB-induced CCAAT/enhancer-binding protein $\delta$ in mouse macrophages, Cell. Signal. 18 (2006) $1492-1500$.

[40] O. Norkina, A. Dolganiuc, T. Shapiro, K. Kodys, P. Mandrekar, G. Szabo, Acute alcohol activates STAT3, AP-1, and Sp-1 transcription factors via the family of Src kinases to promote IL-10 production in human monocytes, J. Leukoc. Biol. 82 (2007) 752-762.

[41] M.S. Vivarelli, D. McDonald, M. Miller, N. Cusson, M. Kelliher, R.S. Geha, RIP links TLR4 to Akt and is essential for cell survival in response to LPS stimulation, J. Exp. Med. 200 (2004) 399-404.

[42] K. Saegusa, S. Yotsumoto, S. Kato, Y. Aramaki, Phosphatidylinositol 3-kinase-mediated regulation of IL-10 and IL-12 production in macrophages stimulated with CpG oligodeoxynucleotide, Mol. Immunol. 44 (2007) 1323-1330.

[43] M. Ohtani , S. Nagai, S. Kondo, S. Mizuno, K. Nakamura, M. Tanabe, T. Takeuchi, S. Matsuda, S. Koyasu, Mammalian target of rapamycin and glycogen synthase kinase 3 differentially regulate lipopolysaccharide-induced interleukin-12 production in dendritic cells, Blood 112 (2008) 635-643.

[44] Gerosa F, Baldani-Guerra B, Lyakh LA, Batoni G, Esin S, Winkler-Pickett RT, Consolaro MR, De Marchi M, Giachino D, Robbiano A, Astegiano M, Sambataro A, Kastelein RA, Carra G, Trinchieri G, Differential regulation of interleukin 12 and interleukin 23 production in human dendritic cells. J Exp Med. 205 (2008) 1447-1461. 


\section{Footnotes}

Abbreviations used in this paper: Ab, antibody; BMDCs, bone marrow-derived dendritic cells; DCs, dendritic cells; ELISA, enzyme linked immunosorbent assay; ERK, extracellular signal-regulated kinase; FADD, Fas-associated death domain protein; FCS, fetal calf serum, FITC, fluorescein isothiocyanate; GM-CSF, granulocyte-macrophage colony-stimulating factor; h, hour; JNK, c-jun N-terminal kinase; IFN, interferon; IL, interleukin; IRF, IFN regulatory factor; LPS, lipopolysaccharide; mAb, monoclonal Ab; MAPK, mitogen-activated protein kinase; MEK, MAPK/ERK kinase; min, minute; MyD88, myeloid differentiation

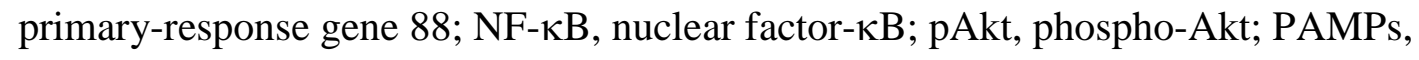
pathogen-associated molecular patterns; P3C, Pam3CSK4; PI3K, phosphatidylinositol 3-kinase; PE, phycoerythrin; PerCP, peridinin chlorophyll protein; pERK, phospho-ERK; pJNK, phospho-JNK; pp38 MAPK, phospho-p38 MAPK; Th1, T helper type 1; TLR, Toll-like receptor; TLR-L, TLR ligands; TNF, tumor necrosis factor; TNFR1, TNF receptor type 1; TNFR2, TNF receptor type 2; TRADD, TNFR1-associated death domain protein; TRAF, TNF receptor-associated factor; TRIF, TIR domain-containing adaptor inducing IFN- $\beta$; WT, wild type. 


\section{Figure legends}

Fig. 1. Cytokine production by WT and TNF- $\alpha^{-/-}$DCs upon TLR stimulation. (A) BMDCs from WT or TNF- $\alpha^{-/-}$mice were stimulated with $1 \mu \mathrm{g} / \mathrm{ml}$ LPS plus $100 \mathrm{ng} / \mathrm{ml}$ P3C (TLR-L) for 24 h. (B) BMDCs from WT or TNF- $\alpha^{-/-}$mice were stimulated with TLR-L in the presence or absence of TNF- $\alpha$ (100 ng/ml) for $24 \mathrm{~h}$. The amount of cytokines in the culture supernatants was measured by ELISA. Each column represents the mean \pm SE of eleven (A) or nine (B) independent experiments. Statistical significance was calculated by Student’s $t$-test (**: $p<0.01 ; * * *: p<0.001$; NS: not significant).

Fig. 2. Expression of surface molecules on WT and TNF- $\alpha^{-/-}$DCs upon TLR stimulation. BMDCs from WT or TNF- $\alpha^{-/-}$mice were stimulated with TLR-L for $24 \mathrm{~h}$. Expressions of CD86, CD40, and I-A ${ }^{\mathrm{b}}$ were analyzed by flow cytometry. (A) Representative histogram of the each molecule on WT and TNF- $\alpha^{-/-}$DCs. (B) Each column represents the mean \pm SE of three independent experiments. MFI, mean fluorescence intensity.

Fig. 3. Activation of ERK1/2, p38 MAPK, JNK1/2, and Akt in TNF- $\alpha^{-/-}$DCs upon stimulation with TNF- $\alpha$ and TLR-L. BMDCs were treated with TNF- $\alpha(100 \mathrm{ng} / \mathrm{ml})$ and/or TLR-L for 7, 15, or 30 min, and whole cell lysates were prepared. Levels of phospho- ERK1/2 (pERK1/2), phospho-p38 MAPK (pp38 MAPK), phospho-JNK1/2 (pJNK1/2), and phospho-Akt (pAkt) in the cell lysates were determined by immunoblotting. GAPDH level was determined as an internal control for each sample. (A) Representative immunoblot is shown. (B) The relative intensity of the specific band is shown. Each column represents the mean \pm SE of three independent experiments. Statistical significance was calculated by Student's $t$-test $(* *$ : 
$p<0.01 ; * * *: p<0.001)$.

Fig. 4. The effect of ERK1/2, p38 MAPK, or PI3K inhibition on IL-10 and IL-12 production by TNF- $\alpha^{-/-}$DCs stimulated with TNF- $\alpha$ plus TLR-L. BMDCs were pretreated with $10 \mu \mathrm{M}$ U0126 (a MEK1/2 inhibitor), 30 M SB203530 (a p38 MAPK inhibitor), $10 \mu \mathrm{M}$ LY294002 (a PI3K inhibitor), or vehicle alone (0.1\% DMSO) for $1 \mathrm{~h}$ and then stimulated with TNF- $\alpha$ (100 $\mathrm{ng} / \mathrm{ml}$ ) plus TLR-L for $24 \mathrm{~h}$ in the presence of each inhibitor. The amount of IL-10 and IL-12 in the culture supernatants was measured by ELISA. Each column represents the mean \pm SE of five independent experiments. Statistical significance was calculated by Dunnett's test (**: $p<0.01)$. 
Fig. 1. Hirata et al.

(A)
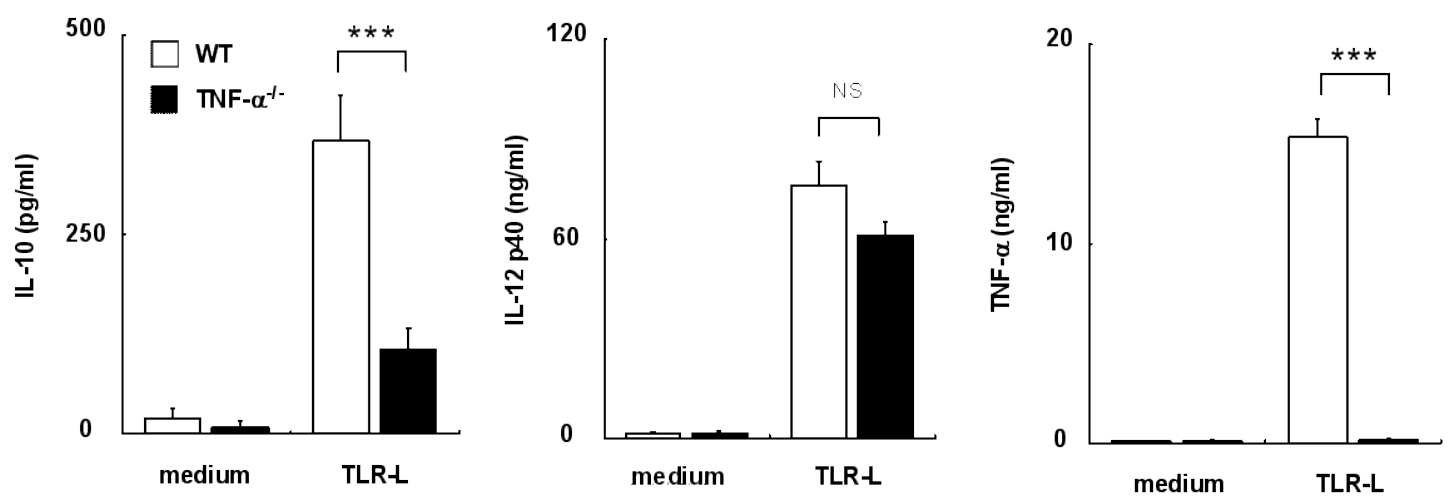

(B)
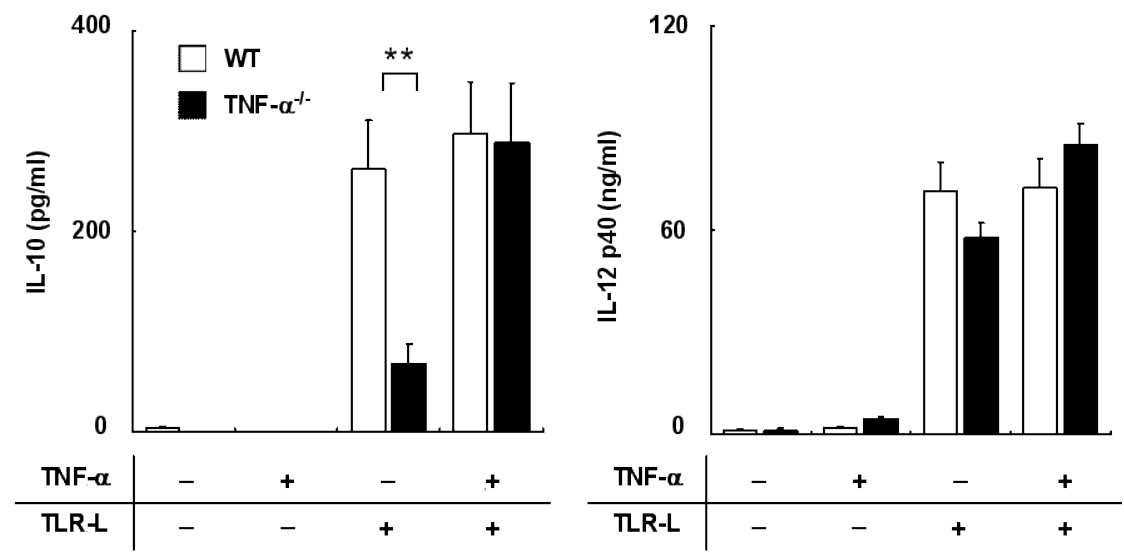
Fig. 2. Hirata et al.

(A)

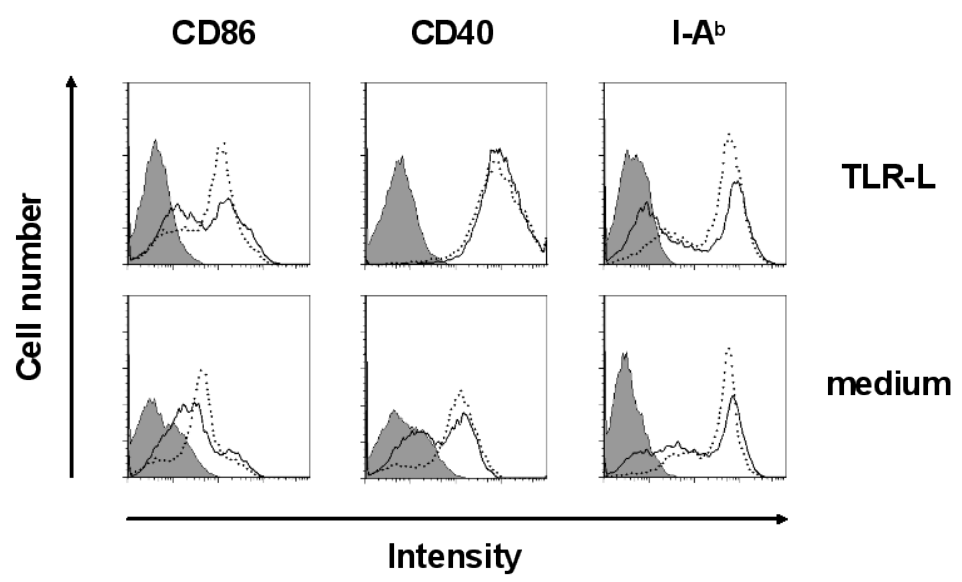

Isotype Control - Specific mAb (WT) $\ldots$ Specific mAb (TNF- $\alpha^{-1-}$ )

(B)

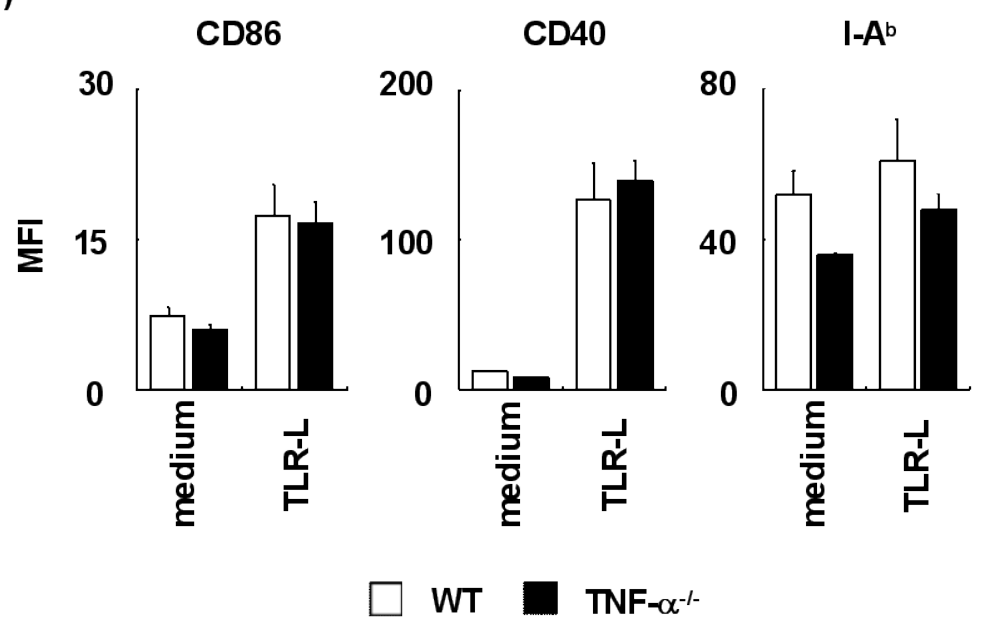


Fig. 3. Hirata et al.

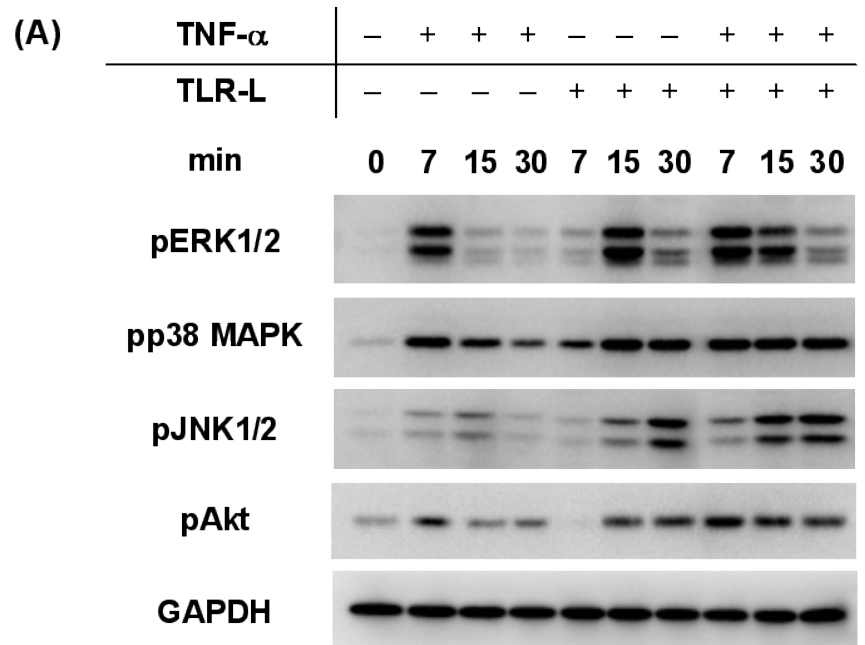

(B)

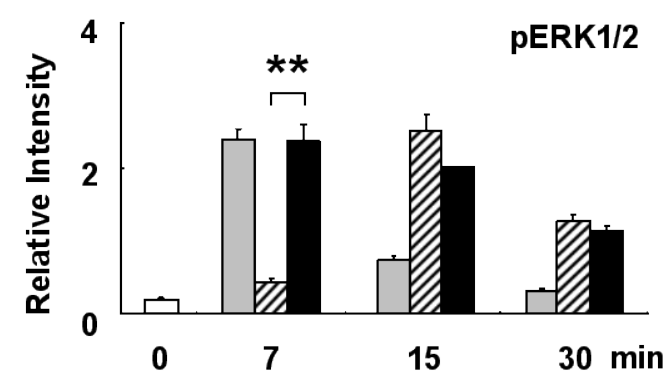

(D)

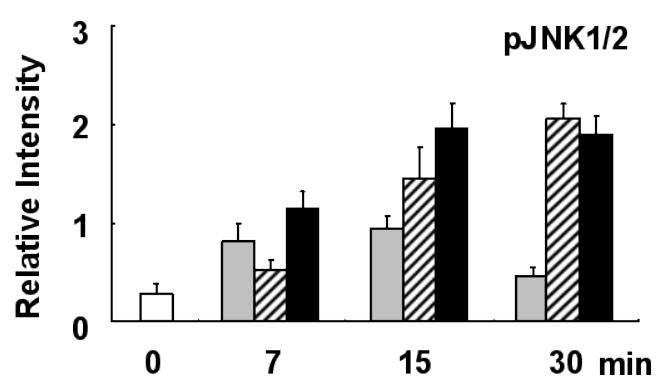

(C)

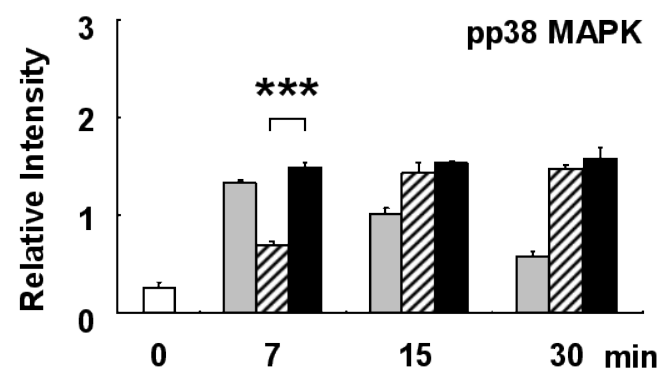

(E)

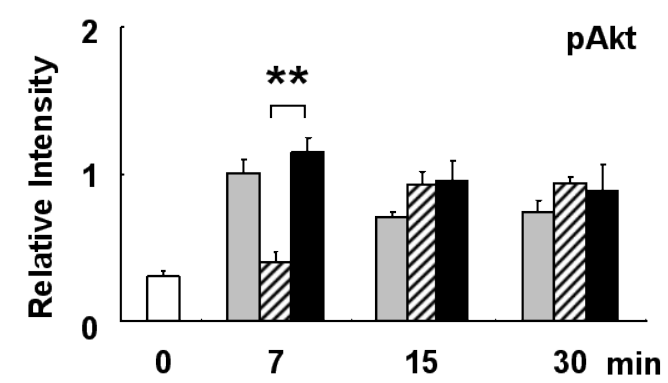

TLR-L $\square$ TNF- $-\alpha+T L R-L$ 
Fig. 4. Hirata et al.
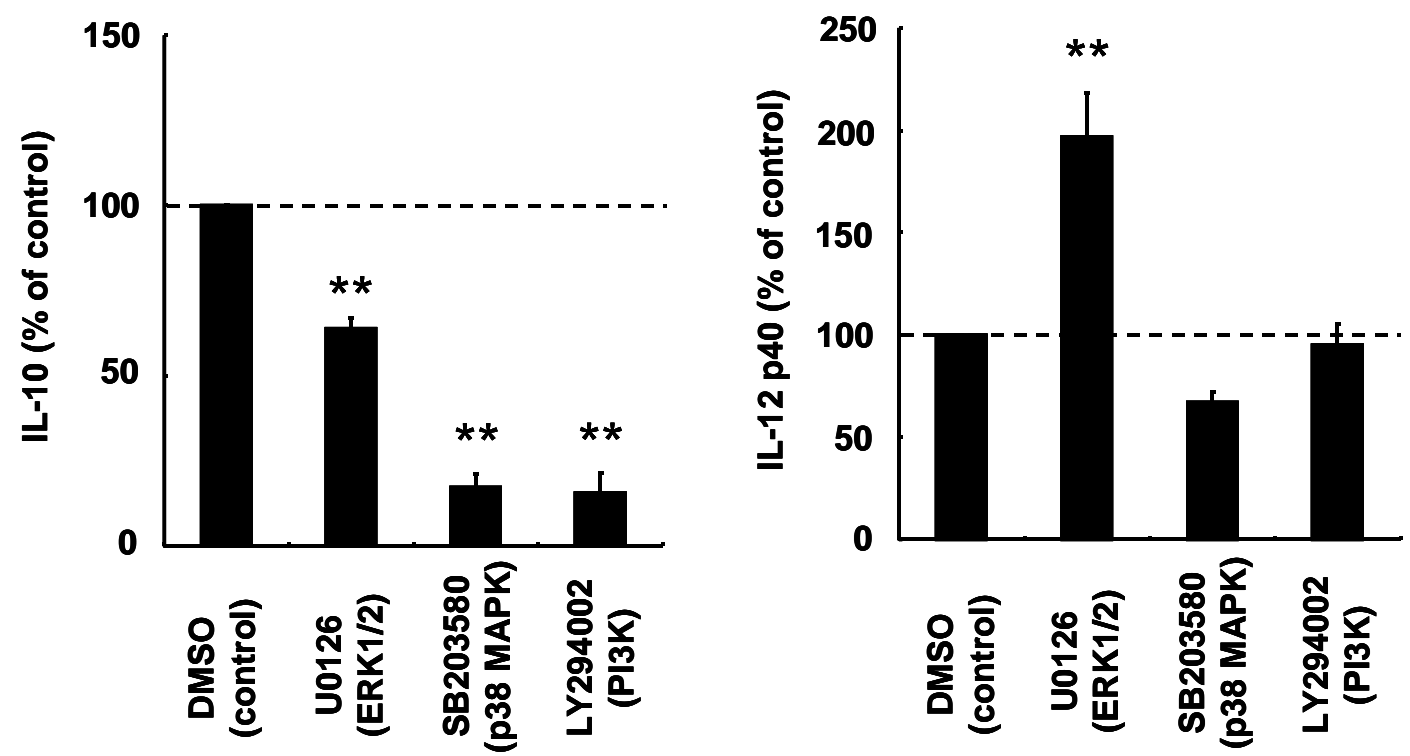